ArtefaCToS. Revista de estudios de la ciencia y la tecnología

eISSN: 1989-3612

Vol. 7, No. 1 (2018), 2a Época, 121-142

DOI: http://dx.doi.org/10.14201/art201871121142

\title{
Conocimiento y Educación Superior desde la perspectiva de género: sociología, políticas públicas y epistemología
}

Knowledge and Higher Education from a Gender Perspective: Sociology, Public Policies and Epistemology

\section{Eulalia PÉREZ SEDEÑO}

Consejo Superior de Investigaciones Científicas

Instituto de Filosofía-Centro de Ciencias Humanas y Sociales

eulalia.psedeno@cchs.csic.es

Recibido: 17/01/2018. Revisado: 19/01/2018. Aceptado: 24/01/2018

\section{Resumen}

En los últimos treinta años se han producido grandes cambios en la Educación Superior y la investigación españolas. Se ha debido a diversas causas, pero, fundamentalmente al aumento de universidades públicas y privadas y a las nuevas políticas públicas que fomentan la investigación y la igualdad, promovidas desde los estudios de género y las organizaciones feministas.

En este trabajo hacemos un somero repaso a estas cuestiones, examinando cómo ha variado la situación de las mujeres en el sistema de Educación Superior y en los Organismos Públicos de Investigación. También se examinan las políticas públicas que pretenden impulsar la igualdad entre mujeres y hombres a través de ciertas leyes. Y se concluye con una reflexión sobre los cambios que todo ello ha supuesto en los contenidos de conocimiento y en la propia noción de ciencia.

Palabras clave: género; carrera investigadora; infrarrepresentación; filosofía feminista de la ciencia. 


\begin{abstract}
In the last thirty years there have been major changes in Spanish higher education and research. It has been due to various causes, but mainly due to the increase of public and private universities and new public policies that stimulate research and equality, all promoted by gender studies and feminist organizations.

In this paper we briefly review these issues, examining how the situation of women in the Higher Education system and in Public Research Organizations has changed. It also examines public policies that seek to enhance equality between women and men through certain laws. And it concludes with a reflection on the changes that all this has brought about in the contents of knowledge and in the very notion of science itself.
\end{abstract}

Keywords: Gender; Research Career; Under-Representation; Feminist Philosophy of Science.

\title{
1. Introducción
}

Hasta el último tercio del siglo XX, la situación de las mujeres en los sistemas de Ciencia y Tecnología y de Educación Superior (CyT y ES) fue escasa en todo occidente, a pesar de que el acceso a las universidades se produce en estos países, por lo general, de forma generalizada en la segunda mitad del siglo XIX. Estados Unidos fue el país pionero en el acceso de las mujeres a la educación superior. La primera universidad que admitió mujeres (no de forma extraordinaria) fue la de Oberlin, fundada en 1833 con otra denominación y ese año se matricularon 15 mujeres. En 1837, ya como Oberlin College, se matricularon 4 mujeres para obtener el grado, de las cuales tres lo obtendrían.

En Iberoamérica, el acceso de las mujeres a los estudios universitarios se produjo a partir de la década de 1860. En España, la primera mujer que se matricula en la Universidad espańola es María Elena Maseras Ribera, en la Facultad de Medicina de la Universidad de Barcelona y en el curso 1872-73. La siguen María Dolores Aleu Riera y Martina Castells Ballespí que se doctoran en medicina en 1882, el mismo ańo en el que se dicta un decreto mediante el cual se limitaba el acceso de las 'señoras' a la Enseñanza Superior, excepto con permiso de la 'autoridad competente'. Hasta el 8 de marzo de 1910 no se eliminó esa restricción en España. Poco después, en 1914, María Sordé Xipell se licencia en Ciencias y en 1917 Catalina de Sena Vives Pieras se convierte en la primera española en conseguir el doctorado en Ciencias ${ }^{1}$.

\footnotetext{
${ }^{1}$ Hay algunas excepciones previas en las aulas universitarias de Salamanca y Alcalá de Henares, en los siglos XV-XVI (Pérez Sedeño y Canales, 2012).
} 
En Brasil, México, Chile, Cuba y Argentina también las mujeres acceden a la educación superior a finales del siglo XIX. Según La Gaceta de México en 1877 obtuvo su título de médica Zenaida Ucounkoff; una década después obtendría su título en medicina Matilde Montoya. También en 1877 Chile permite el acceso a la universidad y, en la siguiente década, Ernestina Pérez y Eloísa Díaz se matriculan en la Facultad de Medicina. Eloísa Díaz se licencia en Medicina y Farmacia en 1886 y obtiene en 1887 el título de Doctora en Medicina y Cirugía. Ese mismo ańo, lograba el título universitario en medicina la brasileña Rita López y, en 1888, la cubana Laura Martínez Carbajal y del Camino López obtiene la licenciatura en Ciencias Físico-Matemáticas. La primera argentina en obtener un título superior en la Facultad de Ciencias Médicas de la Universidad de Buenos Aires, en 1889, fue Cecilia Grierson (Pérez Sedeño y Canales, 2012). Ese mismo año obtiene el título de ingeniería topográfica la salvadoreña Antonia Navarro Huezo (Uribe Valencia, 2017).

\section{Algunos datos}

El último tercio del siglo XX, sin embargo, supuso un gran cambio en la Educación superior y en la investigación en España. Entre los hechos que contribuyeron a este cambio tenemos la creación de nuevas universidades, la promulgación de la denominada "Ley de la Ciencia" en 1986, la incorporación masiva de las mujeres a los estudios universitarios, y la introducción de los estudios de género.

En la exposición de motivos que habían llevado a la formulación de la denominada Ley de la Ciencia de 1986 se hacía hincapié en que era necesario corregir los defectos de nuestros sistemas universitarios y de $\mathrm{I}+\mathrm{D}+\mathrm{i}$ : recursos económicos y humanos insuficientes, desordenada coordinación y gestión de programas, etc. a la vez que se pretendía impulsar institucional y socialmente la investigación en España. De ese modo se intentaba garantizar "una política científica integral, coherente y rigurosa en sus distintos niveles de planificación, programación, ejecución y seguimiento" para conseguir aumentar los recursos necesarios para la investigación de modo que fueran rentables económica, social y culturalmente.

En su momento, la Ley de la ciencia identificó una serie de problemas e indicadores. Por ejemplo, estaba claro que la promoción de la investigación científico-tecnológica en los ańos subsiguientes exigía un aumento del número de investigadores, así como el aprovechamiento de la experiencia de los ya existentes. Sin embargo, a pesar de volcarse en las personas componentes de la comunidad científica no se estaba teniendo en cuenta a las mujeres, no sólo entonces, sino como futuras integrantes de la comunidad científica. Pues los datos de 1986 mostraban de manera clara la pérdida que se producía a partir del doctorado. Si de los estudiantes universitarios que se licenciaban, algo más de la mitad eran mujeres, en el doctorado y en la lectura de tesis de cada diez apenas cuatro lo eran; en el profesorado, las mujeres quedaban reducidas a la cuarta parte y, como colofón, 
en el estamento de más prestigio y poder, que es el de cátedras de universidad, la proporción era de sólo una mujer por cada nueve hombres (en realidad, 0,7 mujeres). La pérdida de mujeres a lo largo de la carrera académica e investigadora se manifiesta perfectamente en la siguiente gráfica popularmente denominada de 'tijera':

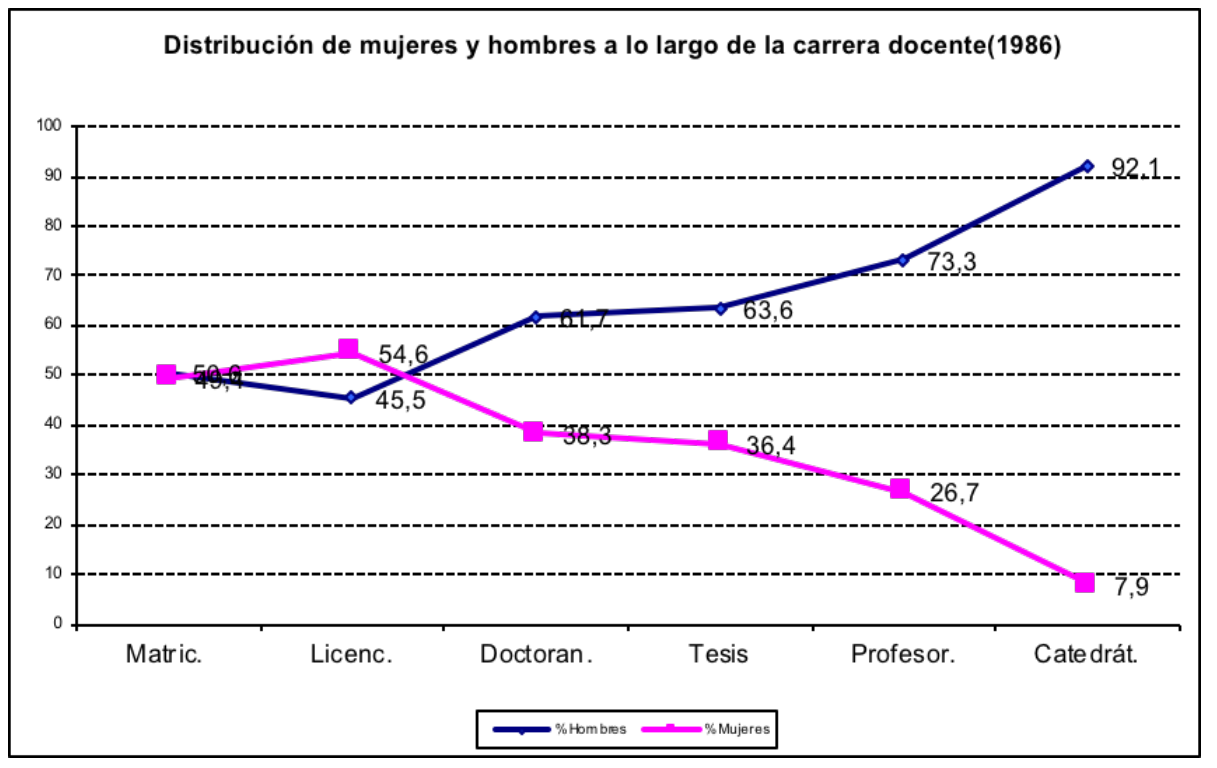

Gráfico 1. Fuente: Pérez Sedeño y Alcalá Cortijo, 2006.

A primeros de los años noventa comienzan a surgir los primeros estudios cuantitativos sobre la situación de las mujeres en la academia española. Dichos estudios ponen de manifiesto la tradicional discriminación jerárquica y territorial. Es decir, las mujeres se quedan en los niveles más bajos del escalafón y en las áreas que tradicionalmente se han considerado 'adecuadas' para ellas (humanidades y áreas biomédicas éstas últimas relacionadas con la esfera del cuidado, tradicionalmente 'femeninas', pero ahora con estatuto universitario). En el curso 1982-83 el porcentaje de mujeres que cursaba carreras de Humanidades era del $64 \%$ y en Ciencias de la Salud ya alcanzaban el $50 \%$.

La voz de alarma surge, sobre todo, por la enorme pérdida de mujeres a lo largo de la carrera docente e investigadora, que ya se había detectado en los ańos ochenta, pero que apenas había suscitado comentarios, excepto entre unas cuantas estudiosas de esta cuestión. Seguramente eso se debía a que nuestro país ha carecido de sensibilidad hacia los problemas de las mujeres, ha habido una enorme ceguera acerca de las consecuencias en todos los terrenos y niveles del desaprovechamiento de recursos humanos y porque ingenuamente se había pensado que el tiempo corregiría esa anomalía. Sin embargo, aunque lentamente, se había iniciado el camino hacia la igualdad. 
Hoy en día, la presencia de mujeres en las instituciones de conocimiento varía según los países y, dentro de éstos, según las áreas y estatuto profesional, pero suele seguir pautas similares. En Espańa, según los últimos datos ofrecidos por el Ministerio de Educación, Cultura y Deporte en el año 2015-2016, el número de mujeres matriculadas en grado eran 705.262 , frente a 581.606 varones, es decir, el 54,8 \% de todas las matrículas en grado. Ese mismo ańo, en máster las mujeres eran $89.593(53,6 \%)$ y los varones 77.315 . Por lo que se refiere al doctorado, en ese mismo año estaban matriculados 24.865 varones y 26.631 mujeres (es decir éstas constituían el $51,7 \%$ de la matrícula).

Los datos más recientes que tenemos con respecto a egresados, procedentes del mismo Ministerio de Educación, nos dicen que en el año 2014/2015 superaron el grado 102.631 mujeres y 65.995 varones (esto es, un 60,8\% de mujeres). En ese mismo ańo superaron el máster 42.308 mujeres y 32.789 varones $(56,3 \%$ mujeres). Incluso en la conclusión del doctorado, paso imprescindible para proseguir una carrera investigadora o en educación superior, y momento en el que en ańos anteriores se iniciaba el dramático descenso del número de mujeres, hoy en día se ha equilibrado: en ese año, 5667 mujeres defendieron con éxito su tesis doctoral y 5649 varones, es decir, las mujeres que concluyeron con éxito su doctorado constituyeron el $50,07 \%{ }^{2}$.

Titulaciones impartidas. Curso 2015/2016

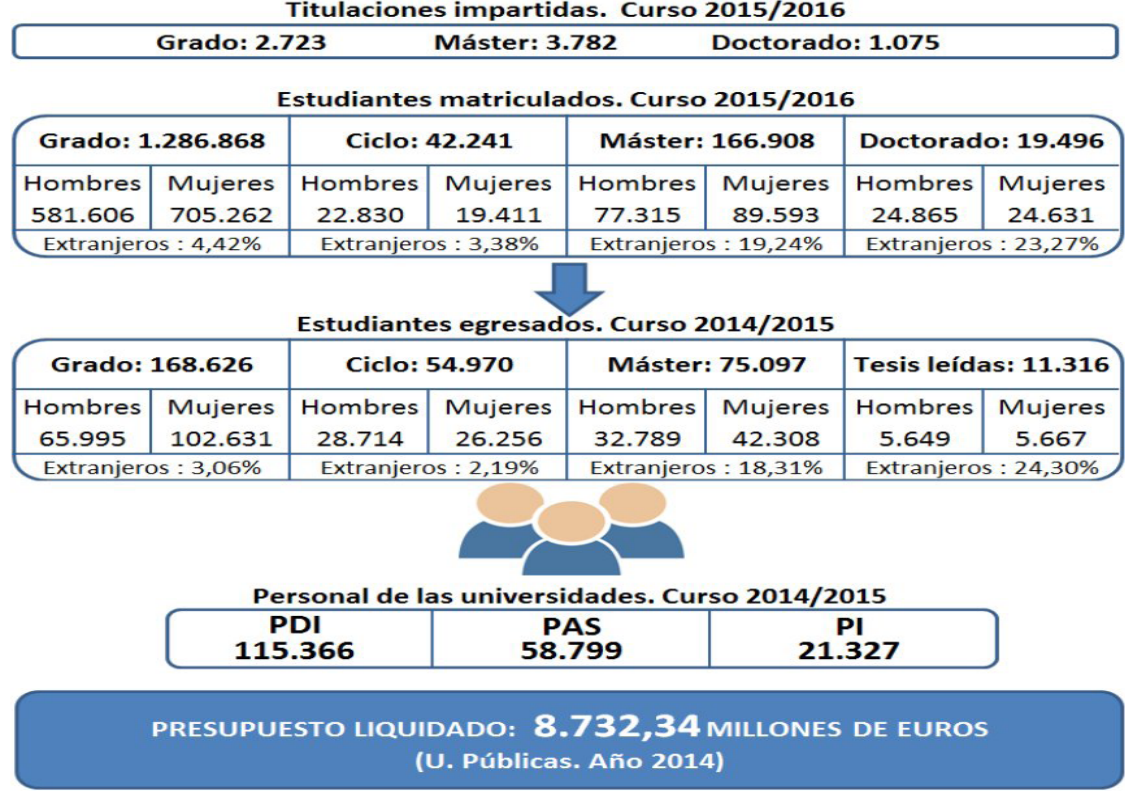

Gráfico 2. Estudiantes matriculados en el curso 2015-2016. Fuente: Web del MECD.

\footnotetext{
${ }^{2}$ Tomado de: http://www.mecd.gob.es/servicios-al-ciudadano-mecd/estadisticas/educacion/universitaria.html. Último aceso, 13 de marzo de 2017.
} 
Como vemos en el gráfico siguiente, extraído del informe Cientificas en cifras 2015, elaborado por la Unidad Mujeres y Ciencia del Ministerio de Economía, Competitividad e Innovación (MINECO), las preferencias de las mujeres están muy repartidas. Como vemos en el ańo 2014/2015 que es el último para el que se presentan datos en este informe, las mujeres se reparten mayoritariamente por el área de ciencias sociales y jurídicas (el 60\%), artes y humanidades (61\%), ciencias de la salud $(72 \%)$, y ciencias $(51 \%)^{3}$. Así pues, las mujeres son mayoría en todas las carreras, excepto en las ingenierías y arquitectura, donde sólo constituyen el $36 \%$ de todo el alumnado ${ }^{4}$.

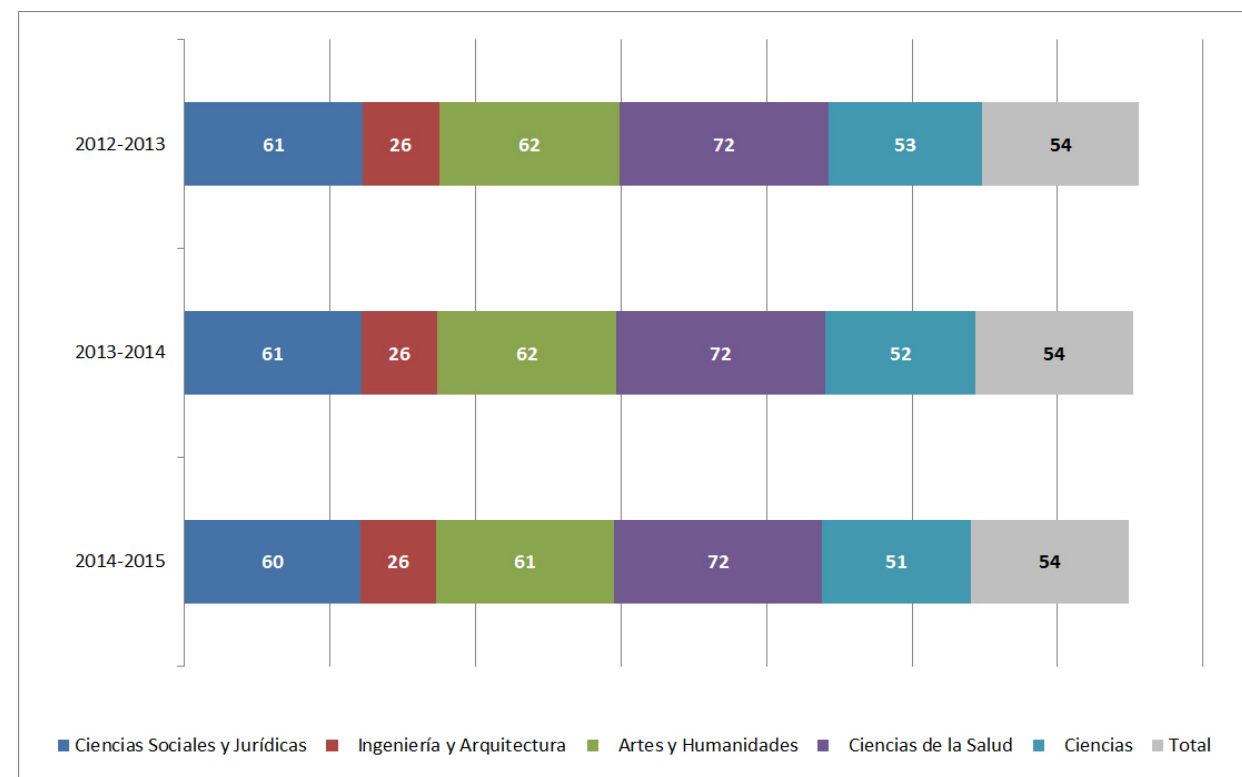

Gráfico 3. Evolución del porcentaje de alumnas matriculadas en Estudios de Grado y Primer y Segundo Ciclo de universidades públicas, según rama de enseñanza. Cursos 2012-13 a 2014-15. (Mujeres sobre el total de cada rama de enseńanza). Fuente: Cientificas en cifras $2015^{5}$.

Esta proporción se mantiene igualmente en las tesis doctorales leídas, pues en casi todas las áreas el porcentaje de mujeres que se doctoranda está en torno al $50 \%$, excepto en las ingenierías y arquitectura, donde sólo alcanzan el $37 \%$. La disparidad entre la matrícula en ingeniería y arquitectura y los doctorados en esta misma área, puede deberse entre otros factores al hecho de que la mayoría de los

\footnotetext{
${ }^{3}$ Este porcentaje está algo distorsionado, pues bajo la rúbrica "ciencias" se incluye la carrera de física, que tan sólo cuenta con un $30 \%$ de mujeres.

${ }^{4}$ Hay que tener en cuenta que esta área agrupa carreras feminizadas, como arquitectura, y otras muy masculinizadas como Ingeniería Naval o Ingeniería Informática.

${ }^{5}$ Debo dar las gracias a la UMYC, en especial a su directora, Ana Puy y a Cecilia Cabello de la FECYT por haberme proporcionado los gráficos en esta resolución.
} 
varones que optan por estas carreras suelen emplearse en el ámbito privado y no se quedan en la Universidad, donde es necesario tener el doctorado para proseguir la carrera académica, pero los ingresos son menores.

Si pasamos a la distribución de mujeres y hombres en la carrera investigadora, encontramos que se sigue manteniendo la tijera de hace ańos (Pérez Sedeño y Alcalá Cortijo, 2006), aunque algo más cerrada. A pesar de que las mujeres, como hemos visto, son mayoría como alumnado matriculado, egresado, en másteres y se igualan en el doctorado. A partir de ahí, comienzan a descender, aunque poco, en los puestos más bajos del escalafón, pero de manera espectacular en la cima de la carrera investigadora, en el caso de las universidades.

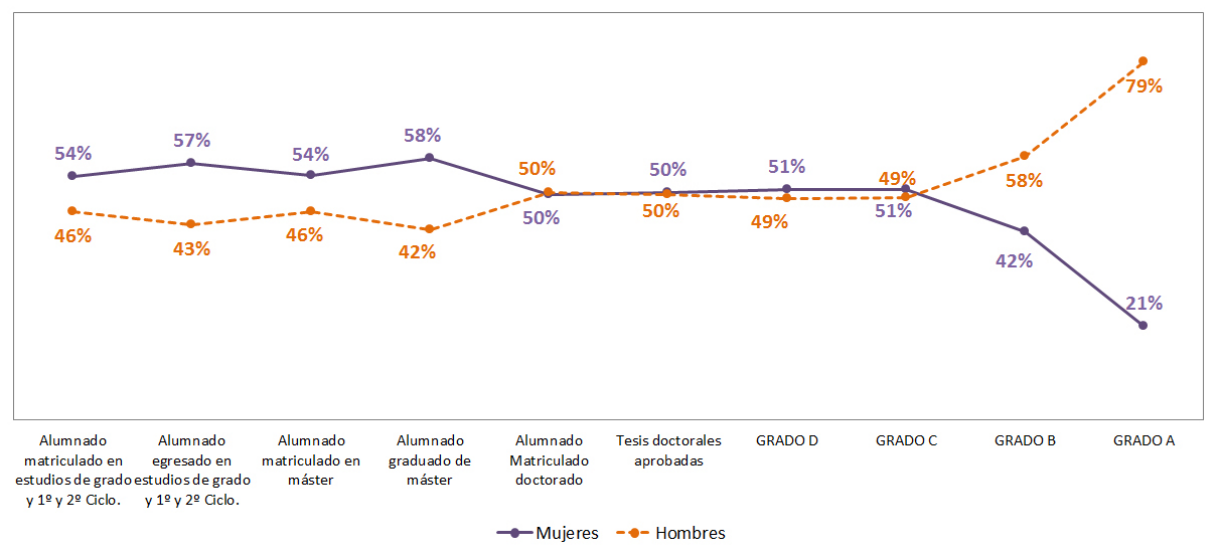

Gráfico 4. Distribución de mujeres y hombres en la carrera investigadora. Universidades públicas. Curso 2014-15. Fuente: Cientificas en cifras 2015.

En los organismos públicos de investigación (OPIs) ${ }^{6}$ podemos decir que el caso es ligeramente peor, puesto que la disminución de mujeres comienza en el primer escalón del escalafón. Como se ve en el gráfico siguiente, las mujeres suponen el 58\% de los contratos post doctorales (Grado D), pero en el caso de los científicos titulares (Grado C), que es el primer puesto fijo en la carrera investigadora de estos organismos, ya descienden al $43 \%$ siendo tan sólo el $25 \%$ como profesoras de investigación (Grado A), el puesto más alto que se puede obtener en estos centros.

\footnotetext{
${ }^{6}$ Los OPIs dependientes de la Secretaría de Estado de Investigación, Desarrollo e Innovación del MINECO son el Consejo Superior de Investigaciones Científicas (CSIC), que es el mayor de todos, Instituto de Salud Carlos III (ISCIII), el Centro de Investigaciones Energéticas, Medioambientales y Tecnológicas (CIEMAT), el Instituto Espańol de Oceanografía (IEO), el Instituto de Astrofísica de Canarias (IAC), el Instituto Nacional de Investigación y Tecnología Agraria y Alimentaria (INIA) y el Instituto Geológico y Geominero de España (IGME).
} 


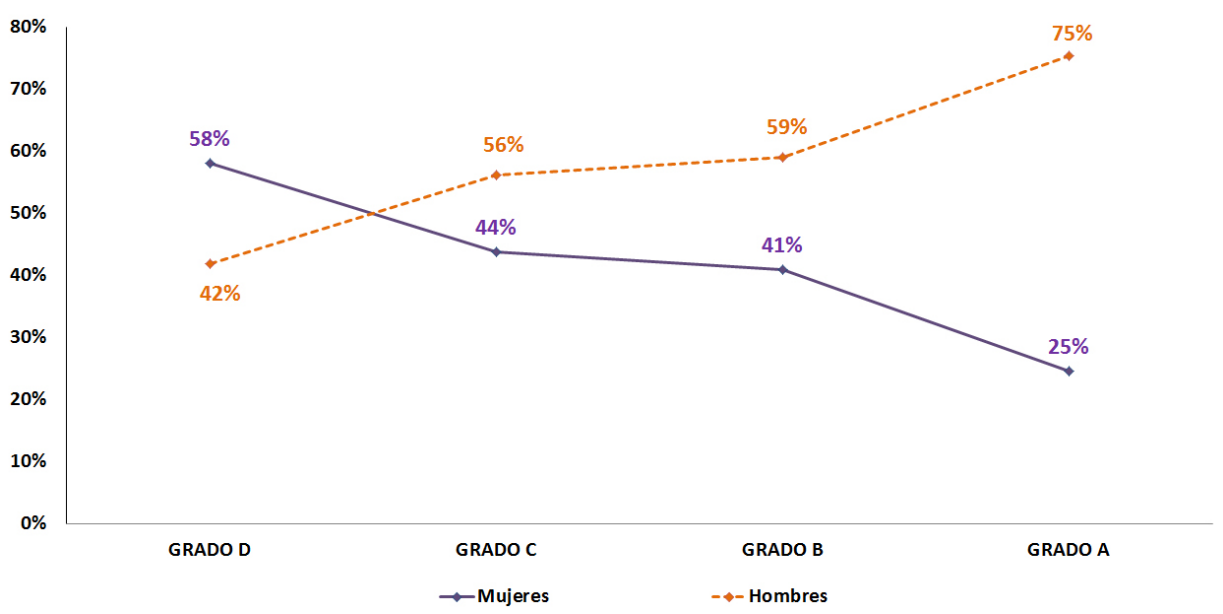

Gráfico 5. Distribución de mujeres y hombres en los OPIs, según categoría investigadora. Fuente: Cientificas en cifras.

Y en los puestos de toma de decisiones la situación es todavía peor: de las 83 universidades públicas y privadas que hay en España, en la actualidad sólo hay 8 rectoras (3 en universidades públicas y 5 en privadas); y, por lo que se refiere a los OPIs acaba de ser nombrada la primera presidenta del CSIC: Rosa Menéndez López es la primera en la historia que preside un OPI.

Estas cifras muestran una serie de problemas. Por un lado, sigue existiendo la denominada discriminación horizontal o territorial, en virtud de la cual las mujeres se van a unas áreas y no a otras. Hace tres o cuatro décadas, esta discriminación horizontal abarcaba a todas las disciplinas científicas, es decir las mujeres eran minoría en todas las carreras de ciencias y de ingenierías (Pérez Sedeño y Alcalá Cortijo, 2006). En la actualidad, como hemos visto son mayoría en todas las disciplinas excepto en las ingenierías; incluso en algunos casos esta mayoría es alarmante, como en ciencias de la salud, dado que la feminización de un área o disciplina conlleva su minusvaloración y a la inversa. Desde luego, la discriminación jerárquica o vertical se sigue dando, aunque no de manera tan virulenta como hace unas décadas en las primeras etapas (Pérez Sedeño y Alcalá Cortijo, 2006), pero sí en los puestos más altos y en los de toma de decisiones.

Una de las cosas que debemos plantearnos ante la lentitud de cambios en las carreras investigadoras de las mujeres es si hay mecanismos que imposibilitan su plena incorporación. Porque además de la discriminación jerárquica o la territorial, hay otros mecanismos más sutiles como el famoso techo de cristal o las microdesigualdades: éstas son esos comportamientos de exclusión, generalmente tan insignificantes, que pasan desapercibidos pero que, al acumularse, crean un clima hostil que disuade a las mujeres a ingresar o permanecer en las carreras científicas y tecnológicas. Las microdesigualdades pueden definirse como el conjunto de comportamientos que tienen por efecto singularizar, apartar, ignorar o descalificar de cualquier modo a un individuo, en función de características in- 
mutables y que no dependen de su voluntad, esfuerzo o mérito, como el sexo, la raza o la edad. Las microdesigualdades crean un entorno laboral y educativo que menoscaba el rendimiento de estos sujetos, porque hace falta tiempo y energía para ignorar este tipo de comportamientos y hacerles frente. A estos mecanismos hay que añadir la falta de corresponsabilidad, el alargamiento de la carrera investigadora y la carencia de mujeres en los puestos de toma de decisiones, así como la falta de perspectiva de género en el diseño de los equipos de investigación que pueden impedir tomar en cuenta diversas cuestiones como la conciliación entre la vida personal y laboral.

\section{Políticas públicas}

El Action Plan of the Women and Science Unit (1999), del V Programa Marco de la Unión Europea (1998-2002) establecía la necesidad de promover la igualdad de género. Esta promoción consistía en fomentar la participación de las mujeres en la producción del conocimiento, incentivando la paridad en la composición de los equipos de investigación e impulsando el liderazgo femenino en proyectos y tipos de investigación (science BY women). De esta manera se intentaba compensar la invisible acción de las mujeres en la ciencia y crear una cultura de trabajo que permitiera el desarrollo de las carreras investigadoras. Asimismo, se animaba a revisar la visión androcéntrica del conocimiento y su producción, que no sólo ha invisibilizado a las mujeres como sujetos de conocimiento, sino que también ha producido un conocimiento construido a partir únicamente de lo masculino (science FOR women). Finalmente, en ese plan de acción se planteaba eliminar el déficit de conocimiento sobre las mujeres y sobre el género y las relaciones de género, haciendo hincapié en que la investigación específica sobre género permite cubrir ciertas lagunas de conocimiento (science $\mathrm{ON}$ women).

Por lo que se refiere a la reforma impulsada por el "Plan Bolonia" para construir un Espacio Europeo de Educación Superior, incorporaba la inclusión de los estudios de género en la docencia y la investigación. En concreto, se establecía la necesidad de formar al alumnado en los valores de igualdad entre mujeres y hombres y no sólo para afrontar los cambios económicos y sociales (Kortendiek, 2011).

Los siguientes programas marco de la UE siguieron apostando por la inclusión de la igualdad de género en el conocimiento. En concreto el VII Programa Marco (2007-2013) desarrolló una serie de acciones para reforzar el papel de las mujeres y la dimensión de género en la investigación. No se trataba tanto de formular políticas que favorecieran que las mujeres entraran y permanecieran en las instituciones científicas (eliminando la fuga de tuberías) sino de implementar políticas que cambiaran las instituciones, mediante la transversalización de la perspectiva de género. 
En el Programa Horizonte 2020 también aparecen reforzadas todas las dimensiones de género en los programas de investigación. Así mismo se pide a las instituciones de investigación que adopten planes de igualdad que identifiquen los sesgos de género y promulguen políticas para erradicarlos. Para ello, las instituciones deben elaborar indicadores que permitan el seguimiento de estas políticas. Con respecto a los proyectos de investigación, se les exige que integren la dimensión de género en todas sus fases: revisión desde la perspectiva de género de las teorías y conceptos utilizados generalmente en su campo científico, planteamiento (si las hipótesis de partida incluyen un análisis de sexo/género que aborde las posibles diferencias o semejanzas que pueda haber entre mujeres y hombres o en animales, tejidos o células), metodología empleada (muestras desagregadas por sexo, objetos de estudio representativos de los diversos sexos/géneros...),experimentación, estrategias de difusión y transferencia de resultados (si facilitan la aplicación a las necesidades específicas de mujeres y hombres), valoración de las consecuencias de la investigación para mujeres y hombres y para la Tierra a largo plazo, etcétera. Además, hay un programa específico, Gender Equality in Research and Innovation (GERI), que canaliza las políticas de igualdad de género en las universidades y los centros de investigación; y el European Research Council (ERC) tiene como uno de sus objetivos primordiales la igualdad de género.

El sistema de vigilancia de la UE (genderwatch) prevé la recogida y difusión de estadísticas, fomenta la participación de las mujeres en los grupos de evaluación y de expertos y promueve la elaboración de estudios que determinen el impacto de género en las distintas ramas de investigación. Además, la UE pública cada tres años She Figures. Statistics and Indicators on Gender Equality in Science, informes donde se presentan datos de todos los países de la Unión Europea. En el año 2006, se creó el European Institute for Gender Equality (EIGE) con el objetivo de difundir la información, intercambiar buenas prácticas y desarrollar instrumentos metodológicos que permitan integrar la dimensión de género en todas las áreas, incluyendo la educación superior y la investigación.

\subsection{Políticas públicas en España}

En España, disponemos de diversas leyes. La Ley Orgánica 1/2004 de Medidas de Protección Integral contra la Violencia de Género, de 28 de diciembre, considera que el sistema educativo es uno de los ámbitos fundamentales para eliminar la violencia contra las mujeres. En concreto, con respecto a la educación superior, el artículo cuatro establece que las universidades fomentarán e incluirán en todos los ámbitos académicos y de manera transversal la formación en igualdad y no discriminación.

Además, disponemos de dos leyes que, si se cumplieran, podrían ayudar de manera drástica a reducir las desigualdades. Nos referimos a la Ley Orgánica 3/2007 para la Igualdad efectiva de mujeres y hombres, de 22 de marzo común- 
mente denominada "Ley de igualdad" y la popularmente llamada "Ley de la Ciencia”, Ley 14/2011 de la Ciencia, la Tecnología y la Innovación, de 1 de junio. Estas leyes fueron precedidas por la Orden del Ministerio de la Presidencia del 8 de marzo de 2005, en la que se adoptaban diversas medidas en los ámbitos del empleo, la investigación y otras medidas en la Administración General del Estado (AGE). Con respecto al empleo y la AGE en el artículo 1.3 se establecía que en ella y en los organismos públicos y empresas dependientes, "los órganos de selección de personal tendrán composición paritaria". En el caso de la investigación se acordaba crear la unidad de "Mujer y Ciencia" (4.1) e incluir, como criterio adicional de valoración en la concesión de ayudas a proyectos de investigación, la participación de las mujeres en los equipos de trabajo (4.2). Así mismo se incluían otras medidas para la igualdad (paridad en los comités de dirección y de expertos y en la composición de los órganos colegiados de la AGE) ${ }^{7}$.

Pero es la Ley de Igualdad la que desarrollaría más completamente estas y otras medidas en todos los ámbitos. En el primer artículo se decía: "Las mujeres y los hombres son iguales en dignidad humana, e iguales en derechos y deberes. Esta Ley tiene por objeto hacer efectivo el derecho de igualdad de trato y de oportunidades entre mujeres y hombres, en particular mediante la eliminación de la discriminación de la mujer, sea cual fuere su circunstancia o condición, en cualesquiera de los ámbitos de la vida y, singularmente, en las esferas política, civil, laboral, económica, social y cultural para, en el desarrollo de los artículos 9.2 y 14 de la Constitución, alcanzar una sociedad más democrática, más justa y más solidaria”. Así, esta ley establecía principios de actuación de los Poderes Públicos y regulaba derechos y deberes de las personas físicas y jurídicas, públicas y privadas, e instauraba medidas destinadas a eliminar y corregir cualquier forma de discriminación por razón de sexo en los sectores público y privado. En concreto, por lo que se refería al ámbito de la ciencia y la educación superior, propugnaba la igualdad de trato y de oportunidades en el acceso al empleo, en la formación y promoción profesionales, y en las condiciones de trabajo. Asimismo, se establecía la transversalidad del principio de igualdad: "Transversalidad del principio de igualdad de trato entre mujeres y hombres. El principio de igualdad de trato y oportunidades entre mujeres y hombres informará, con carácter transversal, la actuación de todos los Poderes Públicos”. La característica de la transversalidad, de llevar la igualdad de género a todos los ámbitos, sitúa al Estado como ente responsable para articular sus recursos y facilitar la igualdad, la participación y la inserción de las mujeres dentro del apartado científico y de investigación.

Además, el artículo 25 dice: "En el ámbito de la educación superior, las administraciones públicas, en el ejercicio de sus competencias, fomentarán la enseñanza y la investigación sobre el significado y el alcance de la igualdad entre mujeres y hombres. En particular, y con tal finalidad, las Administraciones pú-

\footnotetext{
${ }^{7}$ Algunas de estas medidas se proponían en el informe Mujer y Ciencia editado por FECYT en 2004.
} 
blicas promoverán: a) la inclusión, en los planes de estudio en que proceda, de enseñanzas en materia de igualdad entre mujeres y hombres; b) la creación de postgrados específicos; c) la realización de estudios e investigaciones especializadas en la materia”. También en el artículo 77 se crean las Unidades de Igualdad en cada ministerio, encargadas de elaborar estadísticas, estudios que promuevan la igualdad en sus áreas de actividad, asesorar a los órganos competentes en la elaboración del informe sobre impacto por razón de género, fomentar el conocimiento del personal del alcance y significado del principio de igualdad mediante la formulación de propuestas de acciones formativas y velar por el cumplimiento de esta Ley y la aplicación efectiva del principio de igualdad.

La Ley de la Ciencia inserta la perspectiva de género como categoría transversal. En el quinto párrafo del Preámbulo, apartado VIII, el texto legislativo dice: "La perspectiva de género se instaura como una categoría transversal en la investigación científica y técnica, que debe ser tenida en cuenta en todos los aspectos del proceso para garantizar la igualdad efectiva entre hombres y mujeres. Además, se establecen medidas concretas para la igualdad en este ámbito". La inclusión de la perspectiva de género y la noción de transversalidad del principio de igualdad aparece en varias partes de la ley de la ciencia y se establecen actuaciones concretas. Se sitúa la perspectiva de género como categoría en los campos de ciencia, tecnología e innovación, señalando el concepto de transversalidad como herramienta para hacerla efectiva. De forma subsidiaria, en el mismo párrafo, se reclama la presencia equilibrada de mujeres y hombres en todos los ámbitos antes nombrados, haciendo referencia al "I Plan de Igualdad entre mujeres y hombres en la Administración General del Estado y en sus Organismos Públicos”, publicado el 20 de mayo, casi un mes antes que la Ley.

En los "Ejes de actuación" de dicho Plan se señala que la Dirección General de la Función Pública tendrá que hacer un estudio interno anual en relación al acceso al empleo público en el que al menos aparezca una referencia a la existencia de inferior representación femenina o masculina en procesos selectivos (6.2, apartado C). Con ello se pretende estudiar y evaluar el grado de presencia equilibrada de mujeres y hombres, como se refleja en la disposición adicional primera, "Presencia o Composición equilibrada" de la Ley Orgánica de Igualdad entre Mujeres y Hombres: "A los efectos de esta Ley, se entenderá por composición equilibrada la presencia de mujeres y hombres de forma que, en el conjunto a que se refiera, las personas de cada sexo no superen el sesenta por ciento ni sean menos del cuarenta por ciento".

La Ley de la Ciencia va un paso más allá, pues no sólo se habla de la inclusión, sino de la implantación de la perspectiva de género, en su disposición adicional decimotercera, a través de los siguientes 6 puntos que merecen ser expuestos in extenso (pp. 53-54): 
1. La composición de los órganos, consejos y comités regulados en esta ley, así como de los órganos de evaluación y selección del Sistema Español de Ciencia, Tecnología e Innovación, se ajustará a los principios de composición y presencia equilibrada entre mujeres y hombres establecidos por la Ley Orgánica 3/2007, de 22 de marzo, para la igualdad efectiva de mujeres y hombres.

2. La Estrategia Española de Ciencia y Tecnología y el Plan Estatal de Investigación Científica y Técnica promoverán la incorporación de la perspectiva de género como una categoría transversal en la investigación y la tecnología, de manera que su relevancia sea considerada en todos los aspectos del proceso, incluidos la definición de las prioridades de la investigación científico-técnica, los problemas de investigación, los marcos teóricos y explicativos, los métodos, la recogida e interpretación de datos, las conclusiones, las aplicaciones y los desarrollos tecnológicos, y las propuestas para estudios futuros. Promoverán igualmente los estudios de género y de las mujeres, así como medidas concretas para estimular y dar reconocimiento a la presencia de mujeres en los equipos de investigación". En efecto, en los principios de la Estrategia se incluye "La incorporación de la perspectiva de género en las políticas públicas de $\mathrm{I}+\mathrm{D}+\mathrm{i}$ para corregir la pérdida de capital humano asociada a la desigual incorporación de las mujeres y su desarrollo profesional en los ámbitos de la investigación científica y técnica, tanto en el sector público como en el empresarial. Este principio conlleva la incorporación de la perspectiva de género en los contenidos de la investigación científica, técnica y de la innovación para que enriquezca el proceso creativo y la obtención de resultados". Pero no aparece nada más: ni en los objetivos generales, ni en los ejes prioritarios, ni en los mecanismos de articulación. Por lo que se refiere el Plan Estatal de Investigación ${ }^{8}$, la igualdad de género y la perspectiva de género aparece como una de las prioridades del Espacio Europeo de Investigación (p. 5). También afirma que se aplicarán medidas para corregir la brecha de género, que se tendrá en cuenta la dimensión de género en la financiación de actividades $\mathrm{I}+\mathrm{D}+\mathrm{i}$ y se aplicarán criterios estrictos de paridad en las distintas comisiones de evaluación, comités y órganos de gestión y gobernanza (pp.21, 23 y 28). Sin embargo, estos objetivos no se cumplen siempre. Ese es el caso de algunos comités asesores para evaluar la calidad investigadora en la Comisión Nacional de la Evaluación de la Actividad Investigadora (CNEAI): en varios comités de 6 miembros sólo 2 son mujeres (Matemáticas y Física, Ingeniería

${ }^{8}$ Como aparece en las referencias bibliográficas, el documento consultado es el Avance del Plan Estatal. El plan definitivo se publicó el 3 de enero de 2018, cuando este trabajo estaba ya concluido. 
y Arquitectura 1), en otros de 5 componentes sólo hay una mujer (Transferencia de Conocimiento e Innovación) o sólo 2 mujeres de 8 miembros (Ingeniería y Arquitectura 2) ${ }^{9}$. Así mismo entre las prioridades está "incorporar la perspectiva de género, siempre que sea de aplicación, como una variable relevante de carácter transversal en la definición y desarrollo de los contenidos de la investigación" (p. 48; la cursiva es mía y esa coletilla sirve muchas veces de excusa para su incumplimiento).

Siguiendo con los ejes de actuación:

3. El Sistema de Información sobre Ciencia, Tecnología e Innovación recogerá, tratará y difundirá los datos desagregados por sexo e incluirá indicadores de presencia y productividad.

4. Los procedimientos de selección y evaluación del personal investigador al servicio de las Universidades públicas y de los Organismos Públicos de Investigación de la Administración General del Estado, y los procedimientos de concesión de ayudas y subvenciones por parte de los agentes de financiación de la investigación, establecerán mecanismos para eliminar los sesgos de género que incluirán, siempre que ello sea posible, la introducción de procesos de evaluación confidencial.

Dichos procesos habrán de suponer que la persona evaluadora desconozca características personales de la persona evaluada, para eliminar cualquier discriminación por razón de nacimiento, raza, sexo, religión o cualquier otra condición o circunstancia personal o social.

5. La Estrategia Española de Innovación y el Plan Estatal de Innovación promoverán la incorporación de la perspectiva de género como una categoría transversal en todos los aspectos de su desarrollo.

6. Los Organismos Públicos de Investigación adoptarán Planes de Igualdad en un plazo máximo de dos años tras la publicación de esta ley, que serán objeto de seguimiento anual. Dichos planes deberán incluir medidas incentivadoras para aquellos centros que mejoren los indicadores de género en el correspondiente seguimiento anual.

Aunque en el Plan Estatal se dice que "la investigación en materia de género [es] un ámbito específico, así como una variable transversal a incorporar en el desarrollo de las investigaciones orientadas a la búsqueda de soluciones en el resto de los retos de la sociedad" (p. 61), la transversalidad de género no aparece para nada en la Estrategia Nacional.

${ }^{9}$ BOE 21 de febrero de 2017 
Por su parte, la Ley de Ordenación Universitaria (LOU) Ley Orgánica de Universidades 6/2001, de 21 de diciembre, es mucho menos incisiva y ni siquiera se mencionan los estudios de género, quedándose simplemente en los aspectos sociológicos y de inclusión y no discriminación de las mujeres. Por otro lado, la Ley Orgánica 4/2007, de 12 de abril, que modifica la anterior, establece en el Preámbulo que las universidades han de respetar la igualdad entre mujeres y hombres alcanzar la paridad en los órganos de representación y una participación más elevada de las mujeres en los grupos de investigación, recomendando la creación de programas específicos sobre igualdad: "Los estatutos establecerán las normas electorales aplicables, las cuales deberán propiciar en los órganos colegiados la presencia equilibrada entre mujeres y hombres" (cap. 1, art. 13). Lo mismo en el apartado que regula los Órganos de gobierno y representación de las Universidades privadas. Y en las funciones de la Conferencia General de Política Universitaria está promover "que los equipos de investigación desarrollen su carrera profesional fomentando una presencia equilibrada entre mujeres y hombres en todos sus ámbitos" (Artículo 41.4) o en la regulación de la acreditación nacional (art. 57.2) y en la composición de las comisiones de selección de las plazas convocadas (art. 62.3). También declara el derecho de las y los estudiantes a recibir un trato no sexista (art.46.j). Finalmente, en la Disposición adicional duodécima, titulada "Unidades de igualdad" se establece que "Las universidades contarán entre sus estructuras de organización con unidades de igualdad para el desarrollo de las funciones relacionadas con el principio de igualdad entre mujeres y hombres".

Pero la aplicación de estas leyes deja mucho que desear. En primer lugar, no parece haber un seguimiento (o al menos no se ha hecho público) del cumplimiento de estas disposiciones, principios, etc. Además, los valores y necesidades que presiden el diseño del sistema $\mathrm{I}+\mathrm{D}+\mathrm{i}$ perpet $u$ a la orientación masculina tradicional, apenas incorporan los nuevos valores de igualdad o valoración de lo femenino que inspiraron la Ley orgánica de igualdad efectiva de mujeres y hombres de 2007, la Ley integral contra la Violencia de Género (añadida la violencia simbólica) o la Ley de Dependencia, que son los tres grandes marcos legales o de consenso social sobre los que pivota en Espańa el mundo que incluye y valora las mujeres ${ }^{10}$. Por lo general, las leyes o decretos que desarrollan estas leyes generales integran lo políticamente justo y necesario para no ser rechazado en los informes jurídicos previos a su aprobación en las sucesivas comisiones políticas. Estos informes velan porque todo texto normativo o estructural sea coherente con la legislación previa de rango superior y, como es obvio, el campo de aplicación de cualquier "ley orgánica" es tan extenso que no puede faltar una alusión a la perspectiva de género en casi ninguno. Buena prueba de ello son las Conclusiones y Manifiesto del X Encuentro de las Unidades de Igualdad de las Universidades Es-

${ }^{10} \mathrm{El}$ análisis de estas otras dos leyes excede el ámbito de este trabajo. 
pañolas, celebrado en Córdoba el 31 de mayo y 1 de junio de $2017^{11}$. Las barreras obstáculos y resistencias identificadas en diversos ámbitos, y las reivindicaciones siguen siendo, en gran medida, semejantes a las de los primeros encuentros.

\section{Los estudios de género}

Como ya mencionamos, uno de los factores del cambio en Educación Superior e Investigación fue la introducción y desarrollo de los estudios de género o feministas en nuestro país. Esta aparición y evolución de estos estudios en la investigación española responde a dos tipos de intereses: por un parte, la utilidad de promover el análisis de las causas de la persistente desigualdad de las mujeres en todos los ámbitos de la vida pública, incluido el investigador; y por otra, la importancia misma de indagar en las particularidades de la introducción y consolidación de un tipo de trabajo que no encaja fácilmente en la estructura del sistema universitario en el contexto español. Además, las lentes de género o feministas permitieron una nueva mirada sobre supuestos, hipótesis, metodologías, experimentación, en resumen, sobre los contenidos de nuestras teorías y prácticas de conocimiento.

El nacimiento y fuerza actual de los estudios de género a nivel internacional está directamente relacionado con la tercera ola del movimiento feminista de los ańos 60 y $70^{12}$ y con el aumento del número de mujeres investigadoras en esas fechas. Esta combinación dio como resultado una preocupación creciente por el lugar de las mujeres en el mundo académico y por la representación del género en los currículos de enseñanza. El enfoque de género ha tratado, a partir de estas preocupaciones, de indagar en las raíces de la exclusión de las mujeres: recuperando para la historia de la ciencia figuras femeninas silenciadas y olvidadas, analizando las barreras que continúan excluyendo a las mujeres de los ámbitos públicos, planteando la renovación curricular para contribuir a una educación igualitaria, o indagando en los sesgos de género en teorías o disciplinas y en los significados sexuales en el lenguaje y las prácticas de las sociedades humanas ${ }^{13}$.

En definitiva, la discusión feminista sobre los problemas de las mujeres, desde el análisis de su escasez y evolución en los ámbitos de la vida pública hasta las reflexiones teóricas sobre la conceptualización del género y su reflejo en diferentes aspectos del conocimiento y manifestaciones culturales humanas, ha dado lugar al campo de trabajo académico conocido como "estudios de género", "estudios

\footnotetext{
11 Disponible en https://gestioneventos.us.es/_files/_event/_8426/_editorFiles/file/X-Encuentro-UIgualdadEspanolas/X-Encuentro-UIgualdadEspa\%C3\%B1olas_Conclusiones-DEFINITIVAS-y-Manifiesto.pdf. Último acceso 4 de enero de 2018.

${ }^{12}$ Sigo aquí la idea de diversas feministas españolas, que consideran que la primera ola se da en la Ilustración, siendo el feminismo un hijo no querido de la Ilustración (Amelia Valcárcel).

13 Para una introducción a los estudios de género en ciencia y tecnología, véase, por ejemplo, González García y Pérez Sedeño, 2002.
} 
feministas" o "estudios sobre las mujeres". En nuestros días, los estudios de género se han introducido, con distintos grados de institucionalización, en gran cantidad de países, dependiendo las diferencias en su introducción de variables tales como la influencia de los movimientos de mujeres, la existencia de académicas feministas en las Universidades y centros de investigación o de académicos en general dispuestos a introducir temas de género en los currículos, o la flexibilidad de los sistemas universitarios para incorporar asignaturas, programas o departamentos dedicados a temas de género (Wotipka y Ramírez, 2004). Además, son responsables de impulsar y promulgar políticas de igualdad como las expuestas anteriormente.

En el caso español, la introducción de los estudios de género encuentra la resistencia propia de incorporar al sistema ámbitos de trabajo multidisciplinares, para los que no existen departamentos universitarios, áreas de conocimiento, ni titulaciones establecidas ${ }^{14}$. Es, por lo tanto, difícil analizar su introducción, que tiene lugar a través fundamentalmente de programas de postgrado y doctorado promovidos desde algunos departamentos universitarios o desde institutos de investigación de carácter multidisciplinar. En la actualidad, hay 14 másteres de género que se imparten en las siguientes universidades: Universidad de Almería, Universidad Autónoma de Madrid, Universidad Complutense de Madrid, Universitat Jaume I de Castellón, Universidad de La Laguna, Universidad de Murcia, Universidad de Oviedo, Universidad Pablo de Olavide, Universidad del País Vasco/Euskal Erriko Unibersitatea, Universidad de Salamanca, Universidad de Sevilla, Universitat de València y Universidad de Vigo. También hay diversos programas de doctorado, algunos de ellos interuniversitarios, como el que coordina la Universidad Autónoma de Madrid.

Muchos de estos programas de másteres y doctorados se forjaron en seminarios de Estudios de la Mujer. Las Universidades Autónomas de Madrid y de Barcelona fueron las primeras en crear un Seminario de Estudios de la Mujer, ya en 1979. En 1980, con una beca de Antropología como germen, se crea el Seminario de Estudios de la Mujer en la Universidad del País Vasco. En la Universidad de Barcelona, surge en 1982 el Centre d'Investigació Històrica de la Dona (CIHD). Todos ellos respondían a la necesidad de abrir un espacio para transformar el conocimiento sobre las mujeres y el conocimiento mismo, así como para que la universidad y la sociedad evolucionaran (Ballarín Domingo, Gallego Méndez y Martínez Benlloch (1995). Ahora bien, todos ellos se desarrollaban en las áreas de humanidades y ciencias sociales.

Tras los colectivos pioneros, van surgiendo aulas de género, grupos de investigación feminista, seminarios multidisciplinares, algunos de los cuales llegarán a ser Institutos de Investigación, por toda la geografía española. Con propie-

\footnotetext{
${ }^{14}$ El Grado en Igualdad de Género de la Universidad Rey Juan Carlos, el único existente en toda la universidad pública española, fue eliminado en el año 2014.
} 
dad puede decirse que la década de los ochenta vio consolidarse lo que desde el principio mostró una significativa capacidad de desarrollo, consolidación debida muchas veces más al esfuerzo, constancia y buen hacer de las profesoras e investigadoras, unido a la adhesión por parte del alumnado a los nuevos modos de conocer y enseńar, que al respaldo institucional, donde todavía continúan existiendo resistencias. Hoy en día muchos de estos Institutos han arraigado como centros de educación y de investigación.

Como consecuencia de ello surge, en 1996, una convocatoria que se incluye como Programa Sectorial sobre Estudios de las Mujeres y de Género, en el III Plan Nacional de I+D y que a partir del año 2000 pasaría a denominarse Acción Estratégica de Fomento de la Igualdad de Oportunidades entre Mujeres y Hombres, también vinculada al Plan Nacional, a través del área de Socioeconomía. La creación de dicho Programa o Acción Estratégica estaba motivada por la necesidad de formalizar y financiar un área de conocimiento en el que, como ya hemos señalado, se venía trabajando en nuestro país.

El principal objetivo era fomentar la investigación en temas relacionados con la igualdad entre mujeres y hombres que se enmarcaran "en las líneas de investigación propias de los seminarios, institutos, centros y aulas de estudios de las mujeres que incorporan una perspectiva de género en su tarea investigadora”. Las líneas prioritarias incluían pobreza, educación, salud, violencia, trabajo, medios de comunicación y medioambiente. En la actualidad, dentro del programa Retos del Plan nacional de I+D+I hay una subárea temática denominada "FEM Estudios feministas, de la mujer y de género".

\section{Reflexiones finales: una ciencia (y tecnología) por y para las mujeres}

Hemos comentado cómo la UE ya hace años que se plantea eliminar el déficit de conocimiento sobre las mujeres y las relaciones de género, teniendo claro que una investigación específica sobre género permite cubrir ciertas lagunas de conocimiento. En efecto, más allá de los aspectos sociológicos y de igualdad, los estudios de género pretenden ayudar a construir un conocimiento que no discrimine ni pueda ser utilizado para mantener el carácter subordinado y desigual de las mujeres (o de otros grupos marginalizados o vulnerables). Los primeros trabajos se centraron en las áreas de Humanidades y Ciencias Sociales. La historia, la antropología, la sociología, etc. se abordaron con una nueva mirada, reevaluando la aportación de las mujeres a esas disciplinas, sacándolas de la invisibilidad y reformulando nuevas hipótesis y teorías e ideando nuevas metodologías para abordarlas. También se han sacado a la luz y evaluado las metáforas utilizadas, pero también los sesgos metodológicos y androcéntricos en la elaboración de dichas hipótesis o en las prácticas científico-tecnológicas. Poco a poco el interés se fue centrando en otras disciplinas cientificas, en especial en aquellas que se han utilizado y utilizan para mantener el carácter subordinado de las mujeres, esto 
es, las disciplinas biosociales. Y sobre todo se ha criticado una ciencia hecha por los hombres y centrada en ellos como sujetos y objetos de conocimiento. El caso de la biomedicina es especialmente significativo. Esa área se ha fundamentado en la idea de que mujeres y hombres son diferentes, solo en los aspectos reproductivos. Eso ha producido estudios, ensayos clínicos, etc. muy sesgados y que, o se despreciaran otros aspectos no reproductivos de los cuerpos de las mujeres, o que se basaran solo en varones (Valls Llobet, 2008 y García Dauder y Pérez Sedeño, 2017). Por otro lado, hay disciplinas, como la primatología, la biología o la biomedicina, que han incorporado de manera exponencial mujeres que han contribuido con una nueva mirada a la disciplina(García Dauder y Pérez Sedeńo, 2017).

Otra de las áreas reveladoras es la que pretende justificar desigualdades sociales de género basándose en argumentos "científicos" sobre las diferencias innatas entre hombres y mujeres. Se ha escrito mucho sobre el sexismo y el androcentrismo en las teorías científicas del siglo XIX que pretendían demostrar la inferioridad "natural" de las mujeres (fundamentalmente en inteligencia o capacidades), pero desafortunadamente, tales tesis tienen un calado que llega incluso a nuestros días de la mano de algunos socio-biólogos o de psicólogos evolucionistas que presentan el dualismo sexual como rasgo evolutivo y adaptativo de la especie: si somos diferentes por naturaleza, se haga lo que se haga, nada podrá revertirlo. Como seńalaba Edward O. Wilson "Incluso con educación idéntica para hombres y mujeres e igual acceso a todas las profesiones, es probable que los hombres mantengan representación desproporcionada en la vida política, los negocios y la ciencia” (Wilson, 1978, 103). Curiosamente, esta idea es la que cala en el imaginario colectivo, muchas veces a través de los medios de comunicación, pero también de obras literarias o fílmicas, seguramente porque se conforma con la idea profundamente arraigada de diferencias irreconciliables que equivalen, en el fondo a desigualdades: sin embargo, lo opuesto a desigualdad es igualdad -no diferencia- y a ésta última se opone lo idéntico, lo mismo. En definitiva, el gran problema de este tipo de estudios sobre las diferencias sexuales es reducir a dos la gran diversidad y variabilidad humana. Como indica Steven Rose: "si los intentos de contestar estas cuestiones sobre diferencias están plagados de falacias científicas, ¿podrían estas sin embargo tener implicaciones para las políticas públicas haciendo investigación que merezca la pena? La respuesta avanzada a veces es que, si hay tales diferencias y se entienden sus causas, los grupos menos favorecidos podrían ser 'compensados' con alguna forma de educación diferenciada. Pero en la práctica, las afirmaciones de que hay diferencias de inteligencia entre blancos y negros o entre hombres y mujeres siempre se han utilizado para justificar una jerarquía social en la que los hombres blancos continúan ocupando las primeras posiciones (ya sean en economía general o en las ciencias naturales en particular) ... En una sociedad en la que el racismo y el sexismo estuvieron ausentes, la 
cuestión de si blancos u hombres son más o menos inteligentes que los negros y las mujeres simplemente no tendría significado, ni siquiera se preguntaría al respecto" (Rose 2009,788).

Estos análisis han encontrado especial resistencia debido a la falsa creencia de que la ciencia es objetiva, neutra y carente de otros valores que no sean los meramente epistémicos. Pero la irrupción de la teoría feminista, junto con otros enfoques como la filosofía naturalizada de la ciencia, en el análisis y reflexión sobre la ciencia ha proporcionado diversos argumentos en contra de la neutralidad valorativa de la ciencia: como cualquier otra actividad desarrollada por los seres humanos, no se puede entender fuera de su contexto sociocultural. Los argumentos que hacemos a favor de ciertas evidencias dependen del contexto, del mismo modo que consideramos que ciertos datos constituyen evidencia a favor o en contra de cierta hipótesis sólo con respecto a ciertas hipótesis o supuestos previos. Dicho de otro modo, la construcción del conocimiento es una práctica social; por eso, la investigación conlleva necesariamente valores e ideología, pero eso no supone que haya que tolerar de forma indiscriminada las preferencias subjetivas individuales. Lo que confiere objetividad a los productos de la investigación científica es la crítica social más la evidencia empírica. La ciencia es un proceso y actividad de comunidades científicas insertas en contextos sociohistóricos concretos en cuyo seno encontramos valores personales, sociales y culturales, preferencias de grupos o individuales, de tipo cultural, social, que inciden o pueden incidir en diversos modos y grados sobre la práctica científica (Longino, 1990). Esta nueva filosofía feminista de la ciencia considera que los valores contextuales, en concreto los ideológico-políticos, son constricciones relevantes en el razonamiento y la interpretación que conforman el contenido del conocimiento.

Las comunidades epistémicas deben estar abiertas a la crítica, sus componentes tienen igual autoridad epistémica, sin que nadie pueda obligar a los demás a elegir una teoría. Como dice Longino, los individuos tienen autonomía de opinión y es necesario que haya diversidad de perspectivas para que exista "un discurso vigoroso y epistémicamente crítico de verdad" (Longino, 2002, 131 y 154). Ni siquiera el subconjunto de investigadoras que hacen ciencia desde el feminismo tiene una articulación determinada de valores feministas que domina sobre el resto, ya que hay diferentes perspectivas feministas.

La incorporación de pluralidad de perspectivas, especialmente si son socialmente relevantes, conllevará una mayor democratización de la comunidad científica y una ciencia más objetiva, ya que facilitará el cuestionamiento del trasfondo de valores hegemónicos, marcando su carácter político y parcial y anulando sus idiosincrasias particulares (Longino, 1993). Si sólo tenemos la perspectiva de una sola raza, sexo, etc., difícilmente podremos tener una ciencia de miras amplias en la que puedan surgir nuevas preguntas y nuevas soluciones y una nueva forma de hacer ciencia. 
Como se ha señalado en otra ocasión "la objetividad científica y la búsqueda de una mejor ciencia requieren no solo corregir los sesgos de género que se producen en las investigaciones, sino políticas democráticas y participativas en las prácticas científicas comunitarias" (García Dauder y Pérez Sedeño, 2017, 240).

\section{Referencias bibliográficas}

Ballarín Domingo, Pilar, Gallego Mendez, Ma Teresa y Martinez Benlloch, Isabel (1995). Los estudios de las mujeres en las Universidades españolas 197591. Libro Blanco. Madrid: Ministerio de Asuntos Sociales. Instituto de la Mujer.

García Dauder, S. y Pérez Sedeño, Eulalia (2017). Las “mentiras” cientificas sobre las mujeres. Madrid: Los libros de la Catarata.

González García, Marta y Pérez Sedeño, Eulalia (2002). Ciencia, Tecnología y Género. Revista Iberoamericana de Ciencia, Tecnología, Sociedad e Innovación, OEI, 2. http://www.campus-oei.org/revistactsi/numero2/varios2. htm

Kortendiek, Beate (2011). Supporting the Bologna Process by Gender Mainstreaming: A Model for the Integration of Gender Studies in Higher Education Curricula. En Laura Grünberg (ed.), From Gender Studies to Gender IN Studies. Case Studies on Gender-Inclusive Curriculum in Higher Education (pp. 211-228). Bucharest: Unesco - CEPES.

Longino, Helen (1990). Science as Social Knowledge. Princeton: Princeton University Press.

Longino, Helen (2002). The Fate of Knowledge. Princeton: Princeton University Press.

Ministerio de Economía, Competitividad e Innovación (2013). Estrategia Española de Ciencia y Tecnología 2013-2020.

Ministerio de Economía, Competitividad e Innovación (2017). Avance del Plan Estatal de Investigación Cientifica y Técnica y de Innovación 2017-2020. Consulta pública 11 de julio de 2017.

Pérez Sedeño, Eulalia y Alcalá Cortijo, (2006). La ley de la ciencia 20 años después: ¿Dónde estaban las mujeres? Revista madri $+d$, No. Extra 1. Disponible en:

http://www.madrimasd.org/revista/revistaespecial1/articulos/perezalcala. asp 
Pérez Sedeño, Eulalia y Canales Serrano, Antonio (2012). Educación superior e investigación científica: historia, sociología y epistemología. En Capitolina Díaz Martínez y Sandra Dema Moreno (eds.), Sociología y género. Madrid: Editorial Tecnos.

Rose, Steven (2009). Should Scientists Study Race and IQ? No: Science and Society Do Not Benefit. Nature 457 (7231), 786-788.

Unidad Mujeres y Ciencia (UMYC) (2016). Cientificas en cifras 2015, Madrid: MINECO. Disponible en:

http://www.idi.mineco.gob.es/stfls/MICINN/Ministerio/FICHEROS/ Informe_Cientificas_en_Cifras_2015_con_Anexo.pdf

Uribe Valencia, Yamile. (2017) Participación de la mujer en la ciencia en Colombia. Tesis Doctoral dirigida por la Dra. Obdulia Torres en la Universidad de Salamanca.

VallsLlobet, Carme (2008). Mujeres invisibles. Barcelona: Mondadori.

Wilson, Edward O. (1975). Sociobiology: The New Synthesis. Cambridge: Harvard University Press.

Wotipka, Christine Min. y Ramírez Felipe. (2004). A Cross-National Analysis of the Emergence and Institutionalization of Women's Studies Curricula. Contribución presentada en el $99^{\text {th }}$ Annual Meeting of the American Sociological Association, San Francisco. Disponible en:

http://research.allacademic.com/meta/_mla_apa_research_citation/1/0/9/0/9/p109091_index.html?phpsessid=en3slisu6fidg603gl9d11uvm0. 